APS/123-QED

\title{
Chemotactic predator-prey dynamics
}

\author{
Ankush Sengupta, Tobias Kruppa, and Hartmut Löwen \\ Institut für Theoretische Physik II: Weiche Materie, Heinrich-Heine-Universität \\ Universitätsstrasse 1, D-40225 Düsseldorf, Germany
}

(Dated: October 25, 2018)

\begin{abstract}
A discrete chemotactic predator-prey model is proposed in which the prey secrets a diffusing chemical which is sensed by the predator and vice versa. Two dynamical states corresponding to catching and escaping are identified and it is shown that steady hunting is unstable. For the escape process, the predator-prey distance is diffusive for short times but exhibits a transient subdiffusive behavior which scales as a power law $t^{1 / 3}$ with time $t$ and ultimately crosses over to diffusion again. This allows to classify the motility and dynamics of various predatory bacteria and phagocytes. In particular, there is a distinct region in the parameter space where they prove to be infallible predators.

PACS numbers: 05.40.-a,87.17.Jj,05.10.Gg
\end{abstract}

Phagocytes or predatory microbes are hunting their prey by chemotaxis [1, 2], i.e. they sense the concentration of a chemical which is secreted by the prey and is diffusing through the solution [3]. Typically the predator moves along the steepest gradient of the chemical concentration to ultimately find its ejection source. Likewise the prey (for example another microbe) "smells" a secreted chemical from the advancing predator and tries to escape by moving along in the opposite direction of its maximal gradient. This chemotactically coupled predatorprey systems are relevant for many biological microorganisms. In fact, there are many examples of biological relevance for chemotactically coupled predator-prey microorganisms. To name just a few, common microbial predators and phagocytes are Bdellovibrio [4 6], $P$. aeruginosa [7], D. discoideum [8, 9], lymphocytes [10] and $M$. xanthus [11, 12].

Previous theoretical investigations have focussed on spatiotemporal pattern formation in predator-prey colonies 13, 14] which are typically described by nonlinear reaction-diffusion equations [15, 16]. While the latter approaches involve a coarse-grained continuum modelling, there are much less model studies on individual microorganisms. A discrete swarming model of individual self-propelled particles for bacterial colonies has been proposed by Csirok et al [13]. This was elaborated recently by Romanczuk et al [17] based on a related individual model of Schweitzer and Schimansky-Geier [18]. Finally individual autochemotactic models have been studied where the microbe follows its own diffusing secretion [19-22]. In all of these individual models there is no predator involved, apart from a recent study [23] which addressed a lattice model with no chemicals involved.

Here we propose a discrete model which describes both the predator and the prey individually and contains explicitly the diffusion of the two chemicals secreted by the predator and the prey together with the Brownian motion of the latter. The deterministic (fluctuation-free) model is analyzed analytically and by numerical solution which is supplemented by Brownian dynamics computer simulations at finite temperature. Depending on the model parameters and the initial distance between predator and prey, two different dynamical processes are identified which correspond to catching and escaping, and an unstable steady hunting. By analytical treatment, various scaling laws are extracted characterizing and delineating the different regimes. In the absence of noise, the mean-square distance between predator and prey scales with different exponents $\alpha$ as a function of time with a subdiffusive anomalous exponent $\alpha=2 / 3$ for escaping [24] and a ballistic behavior $\alpha=0$ for steady hunting. Brownian motion leads to ultimate diffusion $(\alpha=1)$ such that these exponents are transient. Catching is accompanied by a scaling form of $\left|t-t_{\text {cap }}\right|^{\alpha}$, with $\alpha=2 / 3, t_{\text {cap }}$ being the capture time. In principle, our results allow to map and classify different biological systems into the different regimes.

In our discrete predator-prey model, the predator is at position $\mathbf{r}_{1}(t)$ at time $t$, hunting the prey which is at position $\mathbf{r}_{2}(t)$ and trying to escape. The concentration field of the chemical secreted by the predator (prey) at a constant ejection rate $\lambda_{1(2)}$ is denoted by $c_{1(2)}(\mathbf{r}, t)$. Assuming a gradient sensing scenario for each microbe in response to the chemical secreted by the other, and taking into account effective stochastic fluctuations that are associated with the non-equilibrium self-propulsion mechanism of each, the overdamped equations of motion for the predator and the prey, respectively reads

$$
\begin{aligned}
& \gamma_{1} \dot{\mathbf{r}}_{1}=+\kappa_{1} \nabla c_{2}\left(\mathbf{r}_{1}, t\right)+\boldsymbol{\eta}_{1}(t) \\
& \gamma_{2} \dot{\mathbf{r}}_{2}=-\kappa_{2} \nabla c_{1}\left(\mathbf{r}_{2}, t\right)+\boldsymbol{\eta}_{2}(t)
\end{aligned}
$$

Here, $\gamma_{1(2)}, \kappa_{1(2)}$ and $\boldsymbol{\eta}_{1(2)}(t)$ are the damping constant in the medium, the chemical coupling constant (a measure of the gradient sensing strength) and the effective noise vector associated with stochastic self-propulsion of the predator (prey), respectively. The first term on the right, in both equations, models the systematic contribution of chemotactic response through simple gradient sensing. We take both $\kappa_{1,2}>0$, so that $c_{2}$ acts as a chemoattractant for the predator, while $c_{1}$ is a chemorepellant for the prey. We model $\boldsymbol{\eta}_{i}(t)$ as a Gaussian white noise: $\left\langle\boldsymbol{\eta}_{i}(t)\right\rangle=\mathbf{0},\left\langle\eta_{i \mu}(t) \eta_{j \nu}\left(t^{\prime}\right)\right\rangle=2 \gamma_{i} \beta^{-1} \delta_{i j} \delta_{\mu \nu} \delta\left(t-t^{\prime}\right)$. 
The Greek indices refer to spatial components, while the Roman indices are reserved for the microbe's attributes ( $i=1$ : predator; 2 : prey). Here, $\beta$ corresponds to an inverse effective temperature associated with the stochastic fluctuations, such that $D_{i}=1 /\left(\gamma_{i} \beta\right)$ is the nonchemotactic diffusion constant of the microbe concerned. Hydrodynamic interaction between the microbes is neglected.

The diffusion equation of each of the chemicals reads

$$
\frac{\partial c_{i}(\mathbf{r}, t)}{\partial t}+\mathbf{u}_{i}(\mathbf{r}, t) \cdot \nabla c_{i}(\mathbf{r}, t)=D_{c i} \nabla^{2} c_{i}(\mathbf{r}, t)+\lambda_{i} \delta\left[\mathbf{r}-\mathbf{r}_{i}(t)\right]
$$

where $D_{c i}$ is the diffusivity of the corresponding chemical, and we have assumed each microbe as a point-source emitter. $\mathbf{u}_{i}(\mathbf{r}, t)$ is the advective flow-field set in the medium due to the motion of the microbe. In practice, a microbe has a typical mesoscopic size ' $a$ '. Expressing lengths in units of $a: r \rightarrow r^{\prime \prime}=r / a$, and time in units of $\tau_{0}=a^{2} / D_{c i}: t \rightarrow t^{\prime \prime}=t / \tau_{0}$, Eq.(3) reduces to

$$
\left[\frac{\partial}{\partial t^{\prime \prime}}+\frac{a \mathbf{u}_{i}(\mathbf{r}, t)}{D_{c i}} \cdot \nabla^{\prime \prime}-\nabla^{\prime \prime 2}\right] c_{i}(\mathbf{r}, t)=\frac{a^{2} \lambda_{i}}{D_{c i}} \delta\left[\mathbf{r}-\mathbf{r}_{i}(t)\right]
$$

Simple spatial gradient sensing microbes are known to move slowly with velocity on the order $v \sim 10^{-2}-$ $10^{-1} \mu \mathrm{m} / \mathrm{s}$, and are typically of size $a \sim 1-10 \mu \mathrm{m}$. Chemicals are secreted typically at $\lambda_{i} \sim 10^{3}$ molecules/s and diffuses at $D_{c i} \sim 10^{2}-10^{3} \mu \mathrm{m}^{2} / \mathrm{s}$. Under such practical situations, noting that the magnitude of the flow-field $\left|\mathbf{u}_{i}\right|$ can be at most on the order of $v$, we have the dimensionless factor av $/ D_{c i} \sim 10^{-5}-10^{-2}$. This makes the advective term negligible, and reverting to the original variables: $r^{\prime \prime} \rightarrow r$ and $t^{\prime \prime} \rightarrow t$, Eq.(4) simplifies to

$$
\frac{\partial c_{i}(\mathbf{r}, t)}{\partial t}-D_{c i} \nabla^{2} c_{i}(\mathbf{r}, t)=\lambda_{i} \delta\left[\mathbf{r}-\mathbf{r}_{i}(t)\right]
$$

Fast moving microorganisms with $v \sim 10-10^{2} \mu \mathrm{m} / \mathrm{s}$ (capable of producing appreciable advection in the medium) are known to chemotax by 'temporal sensing' mechanism of the chemical gradient [25] which we do not address here. Also, the detailed influence of the microbe's shape and distribution of chemical sensors on the spatial gradient sensing itself are not considered in our model.

For an unconfined space in three dimensions, the Green's function solution to Eq.(5) yields

$c_{i}(\mathbf{r}, t)=\lambda_{i} \int_{0}^{t} d t^{\prime} \frac{1}{\left(4 \pi D_{c i}\left|t-t^{\prime}\right|\right)^{\frac{3}{2}}} \exp \left(\frac{-\left(\mathbf{r}-\mathbf{r}_{i}\left(t^{\prime}\right)\right)^{2}}{4 D_{c i}\left|t-t^{\prime}\right|}\right)$

Brownian dynamics of the predator-prey system is implemented to simulate the chemotactic motion (eqs.(1) and (2)), and using Eq.(6) to calculate the spatial gradient of the chemical concentration. We measured time in units of $\lambda_{2}^{-1}$, lengths in units of $l_{0}=0.1 \sqrt{D_{c 2} / \lambda_{2}}$ and energy in units of $\epsilon_{0}=\gamma_{2} D_{c 2}$. Real estimate yields $\kappa_{i} \sim 10^{3} \epsilon_{0} l_{0}^{3}$ for Dictyostelium [9] moving at $0.2 \mu \mathrm{m} / \mathrm{s}$ up cAMP gradient of $0.01 \mathrm{nM} / \mu \mathrm{m}$, secreted at $10^{3}$ molecules $/ \mathrm{s}$ with
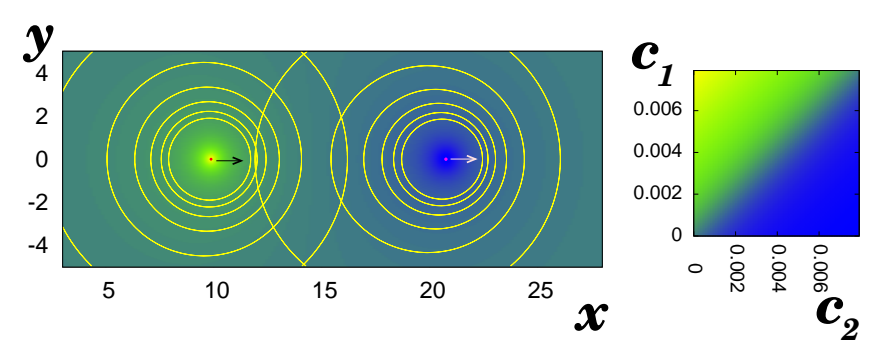

FIG. 1: (color online). (Left) Chemotactic Chase: A predator (red dot on left) chases a prey (red dot on right), while the latter tries to escape through chemotactic gradient sensing of the diffusing chemicals. The arrows indicate their respective direction of motion in absence of fluctuations. The contours around each microbe represent the equi-concentration lines of the secreted chemicals in a two-dimensional projected plane in this case, indicating the asymmetry of the distribution. The color code used here for the spatial distribution of the secreted chemorepellant $\left(c_{1}\right)$ and the chemoattractant $\left(c_{2}\right)$, as they mingle in space, is shown in the right panel.

diffusivity $300 \mu \mathrm{m}^{2} / \mathrm{s}$. Microglial cells [26] moving at $2 \mu \mathrm{m} / \mathrm{min}$ in response to an interleukin gradient of $0.003 \mathrm{nM} / \mu \mathrm{m}$ secreted at 200 molecules $/ \mathrm{min}$ and diffusing at $900 \mu \mathrm{m}^{2} / \mathrm{min}$, has $\kappa_{i} \sim 10 \epsilon_{0} l_{0}^{3}$.

A first look at the problem suggests that the ultimate fate of the prey, i.e. whether it will manage to escape, get captured or be steadily hunted forever, will depend not only on the emission rates and diffusivities of the chemicals, the effective coupling strengths of the microbes to the chemical and their mobilities in the medium, but also on the initial separation between them: $r_{0}=r_{12}(t=0) \equiv\left|\mathbf{r}_{2}(0)-\mathbf{r}_{1}(0)\right|$. Having set the model, it is therefore instructive to examine the zeronoise $\left(\boldsymbol{\eta}_{i}(t)=\mathbf{0}\right)$ deterministic case first, in order to understand the combination of variables relevant in predicting the outcome. Fig.1 shows a simulation snapshot of the chemotactic chase process in the absence of fluctuations. Assuming a steady-state velocity $v_{i}$ for microbe $i$, moving along the $x$-axis, the concentration profile for the chemical secreted by it simplifies to

$$
c_{i}(\mathbf{r}, t=0)=\left(\lambda_{i} / 2 \pi D_{c i} r\right) \exp \left(-v_{i}(x+r) / 2 D_{c i}\right)
$$

In the presence of chemotactic coupling, one then expects $\gamma_{i} v_{i}=\left(1-\delta_{i j}\right) \kappa_{i}\left[\partial c_{j}(\mathbf{r}) / \partial x\right]_{\mathbf{r}=\mathbf{r}_{i j}}$, for each of them. In addition, demanding a condition for steady hunting, whence $v_{i}=v_{j}$, maintaining a constant separation $r_{12}=\Delta$, leads to

$$
\delta\left(\Delta^{*}\right)=\left(1+\Delta^{*-1}\right) \exp \left(-\Delta^{*-1}\right)
$$

Here, $\Delta^{*}=\Delta / \Delta_{0}, \Delta_{0}=\kappa_{1} \lambda_{2} /\left(4 \pi D_{c 1} D_{c 2} \gamma_{1}\right)$ being a length scale, and $\delta=\left(\kappa_{1} \gamma_{2} \lambda_{2} D_{c 1}\right) /\left(\kappa_{2} \gamma_{1} \lambda_{1} D_{c 2}\right)$ will be termed as a sensibility ratio in the predator-prey relationship. Fig.2(a) shows the resulting phase diagram. 


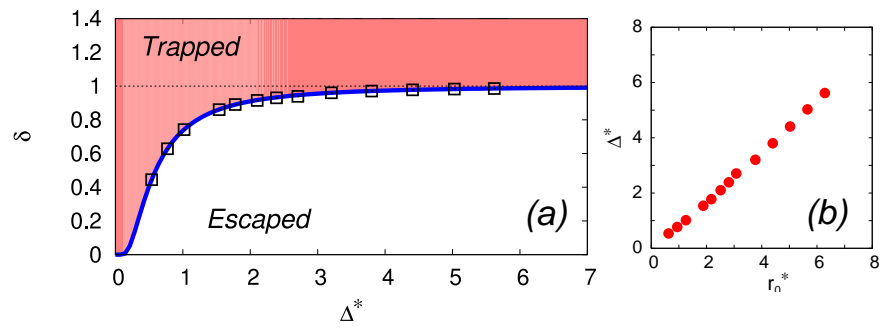

FIG. 2: (color online).(a) Dynamical Phase Diagram of the chemotactic predator-prey system, constructed in the $\Delta^{*}-\delta$ parameter space, showing the trapped (shaded) and escaped phases. The phase boundary (thick solid line) is obtained analytically, and matches with the simulation data (boxes). The horizontal thin dotted line $(\delta=1)$ represents the upperbound for the trapped-to-escaped dynamical phase transition (see text). (b) The dependence of the catching range $\left(\Delta^{*}\right)$ on the initial separation $\left(r_{0}^{*}\right)$, as obtained from simulations.

For a given $\Delta^{*}$, if the sensibility ratio is increased, there is a transition from the free to the trapped phase. To understand this we note that an increase in the chemoattractant coupling or its emission or a decrease of the prey's mobility would prove advantageous to the predator in sensing the prey at a given distance. Also, a decrease in the chemoattractant diffusivity will enable the predator to easily track its prey and ultimately trap it. Similarly, for a given $\delta$, increase in the separation distance will be advantageous to the prey in escaping. Further, since $\Delta>0$ (predator follows prey), the validity of Eq.(8) requires $\delta<1$. This means the phase boundary between the trapped and escaped state lies below the $\delta\left(\Delta^{*}\right)=1$ line in the parameter space. For $\delta \geq 1$, there is no escape. Whatever is the separation between the microbes, the predator will ultimately capture the prey in this case.

In our simulations, the control parameter is the initial distance $r_{0}$ between predator and prey. By tuning $\delta$ for a given $r_{0}$, we actually found the point of transition from trapped to escaped state. The border-line case of steady hunting was found to be unstable. Close to the border-line situation, the predator-prey distance $r_{12}$ remains constant for long times before fluctuations throw them into either the trapped or the escaped state. This constant distance, if identified with $\Delta$, matches the phase boundary (Eq.(8)) perfectly, and $\Delta^{*}$ depends on $r_{0}^{*}\left(=r_{0} / \Delta_{0}\right)$ in a roughly linear fashion (Fig.2(b)).

What are the dynamical features of the escaped and the trapped phases? Our simulations show that for escape (Fig.3(a)), the mean squared displacement of the prey w.r.t. the predator gradually deviates from the initial value $r_{0}$ and grows subdiffusively with time as $\overline{\mathbf{r}_{12}{ }^{2}} \sim t^{\alpha}$, with an exponent $\alpha=2 / 3$, where the bar denotes an averaging over noise for a given $r_{0}$. This behavior finally crosses over to diffusion, $\overline{\mathbf{r}_{12}{ }^{2}} \sim t$, for long times. The crossover time $t_{c o}$ decreases with increasing fluctuation strength $\beta^{-1}$. Zero-noise simulations show the subdiffusive motion as the final long-time behavior, with the same exponent $\alpha$ (Fig.3(b)). This means that
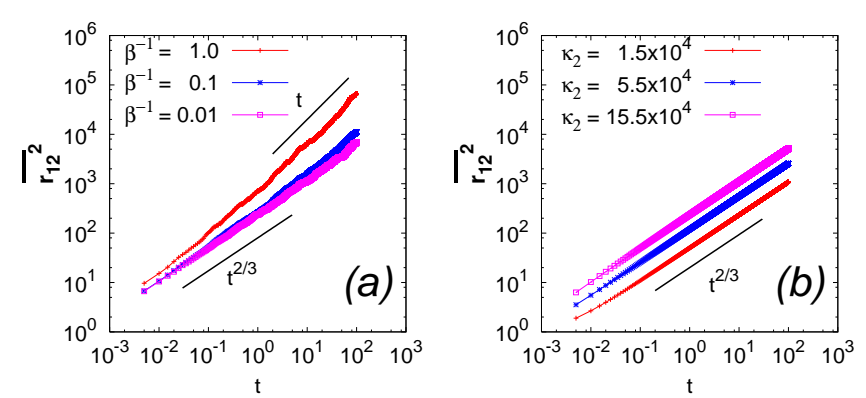

FIG. 3: (color online). Mean square displacement of the prey w.r.t. the predator as a function of time, with $\lambda_{1}=1, \gamma_{1}=$ $10, \gamma_{2}=0.01, D_{c 1}=10^{3}, D_{c 2}=10^{2}, \kappa_{1}=10^{4}$ for (a) $\beta^{-1}=0.01,0.1,1.0$ and $\kappa_{2}=15.5 \times 10^{4}$; and (b) zero noise case $\left(\beta^{-1}=0\right)$ and $\kappa_{2}=15.5 \times 10^{4}, 5.5 \times 10^{4}, 1.5 \times 10^{4}$. The power law behaviors $t^{2 / 3}$ and $t$ are illustrated by the corresponding reference lines drawn.

within this phase, the hunting process continues with a subdiffusive dynamics of the prey in the comoving frame of the predator; but finally due to effects of fluctuation, the prey diffuses away freely. It is therefore appropriate to look for a theoretical estimate of $\alpha$ within the noise-less ideal case. We note from Eq.(7) that at the advancing predator position, the chemo-attractant profile behind the prey is of the form $c_{2}(|x|)=\lambda_{2} /\left(4 \pi D_{c 2}|x|\right)$ in the steady state condition, since $x<0$. Solving the resulting equation of motion in steady state, $\gamma_{1} \dot{x}=\kappa_{1}\left|\nabla c_{2}(x)\right|$, gives $x^{2} \sim t^{2 / 3}$ explaining the subdiffusive exponent. When the fluctuations ultimately overcome the chemotactic coupling, crossover to final diffusion results, requiring $\beta^{-1}=\kappa_{1} c_{2}(|x|)=\kappa_{1} \lambda_{2} /\left(4 \pi D_{c 2}|x|\right)$. At the crossover point from subdiffusive to the diffusive regime, $x\left(t=t_{c o}\right) \sim t_{c o}{ }^{1 / 3}$; implying that the crossover time scales with the inverse effective temperature as $t_{c o} \sim \beta^{3}$.

At the front of each microbe, the respective chemical profile decays much faster $\sim \frac{1}{x} \exp \left(-v_{i} x / D_{c i}\right)$. The predator is thus always at an advantage of sensing the prey from much longer distances. Therefore, for very low $\delta$, a small increase in $\delta$ greatly increases the catching range for the predator. This accounts for the almost vanishing slope of the phase boundary for low $\delta$ in the dynamical phase diagram (Fig.2(a)). In this part of the phase diagram, for two predators with close sensibility ratio, the one with the slightly larger $\delta$ will successfully trap preys which started at a much larger initial separation. For intermediate values of $\delta$, the catching range $\Delta^{*}$ increases at a slower rate with increase in $\delta$. This is because the initial separation is large enough that small changes in $\Delta^{*}$ do not appreciably increase the chemoattractant gradient $\left(\sim 1 / x^{2}\right)$ for the predator. One then needs to considerably alter the sensibility ratio for obtaining a significant change in the chemotactic coupling. As $\delta$ is further increased, $\Delta^{*}$ increases to diverge at $\delta=1$.

For the trapping situation we find a broad skewed distribution $\left(P\left(t_{c a p}\right)\right)$ of capture time $t_{c a p}$, for a fixed $\beta, r_{0}$ and $\delta$ (Fig.4(a)). The mean capture time $\bar{t}_{c a p}$ is, however, 

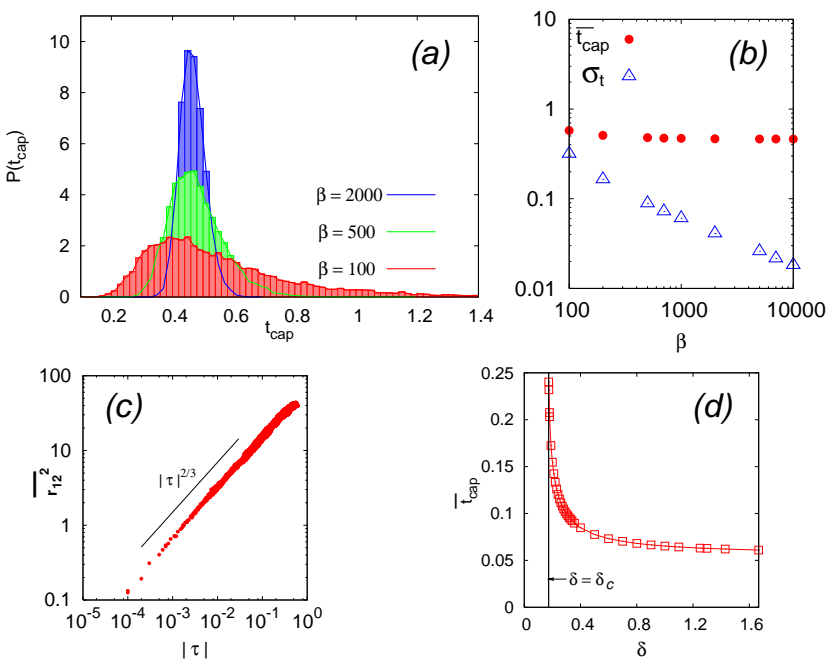

FIG. 4: (color online). (a) Probability distribution of the capture time for the typical values of $\delta$ and $\Delta^{*}$ inside the trapped phase, for $\beta=100,500,2000$. (b) Mean capture time $\left(\bar{t}_{\text {cap }}\right)$ and variance $\left(\sigma_{t}\right)$ of the capture time, as a function of $\beta$. (c) Mean square predator-prey separation $\left(\overline{\mathbf{r}_{12}{ }^{2}}\right)$, close to trapping situations, as a function of the time interval $|\tau|=$ $\left|t-t_{\text {cap }}\right|$. The thick reference line indicates $\tau^{2 / 3}$ power-law behavior. (d) Divergence of the capture time with decrease in $\delta$, for a fixed catching range $\left(\Delta^{*}\right)$, as the trapped-to-escape transition $\left(\delta=\delta_{c}\right.$, shown by the vertical line) is approached.

independent of the effective fluctuation strength, while the variance $\sigma_{t}=\left[\overline{\left(t_{c a p}-\bar{t}_{c a p}\right)^{2}}\right]^{1 / 2}$ decreases with increasing $\beta$ (Fig.4(b)). The trapping dynamics also show non-trivial power-law behavior, very close to capture: $\overline{\mathbf{r}_{12}}(\tau \rightarrow 0-) \sim|\tau|^{2 / 3}$, where $\tau=t-t_{\text {cap }}$ (Fig.4(c)). We note that initially, close to $t=0$, when the predatorprey distance is $\sim r_{0}$, fluctuations dominantly control the individual microbe's motion until a steady chemical concentration profile sets up in the process to favour a systematic dynamics. This time scale is dependent on the individual diffusivities of the chemical: higher the chemical diffusivities compared to the non-chemotactic diffusivities of the microbes, the faster will the predatorprey chemotactic systematics set in. For the trapping dynamics very close to capture, therefore, the predator is already responding to the steady chemoattractant gradient $\gamma_{1} \dot{x}=\kappa_{1}\left|\nabla c_{2}(r)\right|_{r=x} \sim 1 / x^{2}$. Thus, integrating from $x=x(t)$ to $x=x\left(t=t_{\text {cap }}\right)=0$, we obtain the scaling form $x^{2} \sim\left(t_{c a p}-t\right)^{2 / 3}$, with $t<t_{c a p}$. For fixed initial separation, $t_{c a p}$ diverges on approaching from above the corresponding critical value of the sensibility ratio $\left(\delta_{c}\right)$ for escape (Fig.4(d)).

In conclusion, we studied the dynamics of simple gradient sensing chemotactic microorganisms in a predatorprey relationship. Although prokaryotes like most bacteria chemotax chiefly through temporal comparison of chemical gradients, the direct spatial sensing which we studied here is prevalent among eukaryotes like amoeba, yeast cells, neutrophils, lymphocytes and glial cells. Here we have been able to delineate power law behaviors in the chasing process both from theory and numerical simulations, and crossover time scaling with fluctuation strength. A dynamical phase diagram has been obtained to identify conditions for escape and catching, with a border-line unstable steady-hunting situation. A broad class of microbes varying widely with respect to their mobility, secretion rates and diffusivities of ejected chemicals and strength of spatial gradient sensing of chemicals can be located in the phase diagram. Interestingly, a simple sensibility ratio resulting from our model calculation allows a simple criterion to predict the outcome of such hunting processes: a trapped or an escaped situation depending on the initial predator-prey separation, and a no escape situation independent of their initial separation.

We thank S. van Teeffelen, P. Romanczuk, P.S. Hammond and R. Winkler for helpful discussions. This work was supported by DFG within SFB TR6 (project D3).
[1] M. Kollmann et al., Nature 438, 504 (2005).

[2] U. B. Kaupp et al., Annu. Rev. Physiol. 70, 93 (2008).

[3] W. C. K. Poon, in Soft Matter: From Synthetic to Biological Materials (Forschungszentrum Julich GmbH, Germany, 2008), vol. D11, 1-15 of Lecture notes of the 39th IFF Spring School.

[4] M. E. Nunez et al., Colloids Surf., B 42, 263 (2005).

[5] E. Strauch et al., Future Microbiol. 2, 63 (2007).

[6] M. Dori-Bachash et al., Appl. Environ. Microbiol. 74, 7152 (2008).

[7] J. Kato et al., J. Biosci. Bioeng. 106, 1 (2008).

[8] M. Clarke and L. Maddera, Eur. J. Cell Biol. 85, 1001 (2006).

[9] R. Endres and N. Wingreen, PNAS 105, 15749 (2008).

[10] S. Zigmond, J. Cell Biol. 75, 606 (1977).

[11] D. P. Astling et al., Mol. Microbiol. 59, 45 (2006).

[12] J. E. Berleman et al., PNAS 105, 17127 (2008).

[13] A. Csirok et al., Phys. Rev. E 54, 1791 (1996).
[14] M. E. Boraas et al., Evol. Ecol. 12, 153 (1998).

[15] E. F. Keller and L. A. Segel, J. Theor. Biol. 30, 225 (1971).

[16] M. A. Tsyganov et al., Phys. Rev. Lett. 91, 218102 (2003).

[17] P. Romanczuk et al., Eur Phys J. 157, 61 (2008).

[18] F. Schweitzer and L. S.-Geier, Physica A 206, 359 (1994).

[19] Y. Tsori and P.-G. de Gennes, Europhys. Lett. 66, 599 (2004).

[20] R. Grima, Phys. Rev. Lett. 95, 128103 (2005).

[21] R. Grima, Phys. Rev. E 74, 011125 (2006).

[22] A. Sengupta et al., Phys. Rev. E 80, 031122 (2009).

[23] G. Oshanin et al., PNAS 106, 13696 (2009).

[24] For anomalous diffusion in active particles, see e.g.: R. Golestanian, Phys. Rev. Lett. 102, 188305 (2009).

[25] J. Segall et al., PNAS 83, 8987 (1986).

[26] M. Luca et al., Bull. Math. Biol. 65, 693 (2003). 